\title{
Photometric properties and origin of bulges in SB0 galaxies
}

\author{
J. A. L. Aguerri ${ }^{1}$, N. Elias-Rosa ${ }^{2}$, E. M. Corsini ${ }^{3}$, and C. Muñoz-Tuñón ${ }^{1}$ \\ 1 Instituto de Astrofísica de Canarias, Calle via Lactea s/n, 38200 La Laguna, Spain \\ e-mail: jalfonso@ll.iac.es \\ 2 INAF - Osservatorio Astronomico di Padova, vicolo dell'Osservatorio 5, 35122 Padova, Italy \\ 3 Dipartimento di Astronomia, Università di Padova, vicolo dell'Osservatorio 2, 35122 Padova, Italy
}

Received 28 July 2004 / Accepted 11 December 2004

\begin{abstract}
We have derived the photometric parameters for the structural components of a sample of fourteen SB0 galaxies by applying a parametric photometric decomposition to their observed $I$-band surface brightness distribution. We find that SB0 bulges are similar to bulges of the early-type unbarred spirals, i.e. they have nearly exponential surface brightness profiles $(\langle n\rangle=$ $1.48 \pm 0.16)$ and their effective radii are strongly coupled to the scale lengths of their surrounding discs $\left(\left\langle r_{\mathrm{e}} / h\right\rangle=0.20 \pm 0.01\right)$. The photometric analysis alone does not allow us to differentiate SB0 bulges from unbarred S0 ones. However, three sample bulges have disc properties typical of pseudobulges. The bulges of NGC 1308 and NGC 4340 rotate faster than bulges of unbarred galaxies and models of isotropic oblate spheroids with equal ellipticity. The bulge of IC 874 has a velocity dispersion lower than expected from the Faber-Jackson correlation and the fundamental plane of the elliptical galaxies and S0 bulges. The remaining sample bulges are classical bulges, and are kinematically similar to lower-luminosity ellipticals. In particular, they follow the Faber-Jackson correlation, lie on the fundamental plane and those for which stellar kinematics are available rotate as fast as the bulges of unbarred galaxies.
\end{abstract}

Key words. galaxies: bulges - galaxies: elliptical and lenticular, $\mathrm{cD}$ - galaxies: fundamental parameters galaxies: photometry - galaxies: structure

\section{Introduction}

Stellar bars are fairly ubiquitous in galactic discs. They have been found in a large fraction of spiral galaxies (Eskridge et al. 2000) and in almost all Magellanic-type galaxies (Odewahn 1996). The presence of a bar affects the morphological, dynamical and chemical evolution of the host galaxy.

In particular, gas flows and the secular evolution of barred galaxies have been claimed to be viable mechanisms leading to the formation and/or growth of the bulge and have been studied by means of numerical simulation for a long time (see Friedli 1999, for a review).

Numerical simulations have shown that gravitational torques from bars are very efficient at driving interstellar gas toward galactic centres. The main consequences of this gas accumulation is the triggering of bursts of circumnuclear star formation and the formation of a central mass concentration, such as compact bulges, which may weaken or dissolve the bar (Pfenniger \& Norman 1990; Hasan \& Norman 1990). The efficiency of the bar destruction depends on both the concentration of the central component and its growth rate (e.g., Bournaud \& Combes 2002; Shen \& Sellwood 2004). Besides bar-driven accretion, external processes, including satellite accretion (Pfenniger 1991; Aguerri et al. 2001) and galaxy merging (Barnes \& Hernquist 1991), are also able to build a central mass reservoir which makes the bulge grow and the bar dissolve.

Moreover, according to the results of $N$-body simulations, the inner parts of a bar inflate after a few bar rotations because of large-scale violent bending instabilities and settle with an increased thickness and vertical velocity dispersion (e.g., Combes \& Sanders 1981; Combes et al. 1990; Raha et al. 1991). This leads to the establishment of the connection between the bar-buckling mechanism and the formation of boxy/peanut bulges (Bureau \& Freeman 1999; Bureau \& Athanassoula 1999; Chung \& Bureau 2004). The buckling instability does not destroy the bar and forms a central stellar condensation reminiscent of the bulges of late-type spirals (Debattista et al. 2004). This result is in agreement with the early findings of Hohl (1971).

It has been inferred that all the above processes increase the bulge-to-disc ratio, driving the evolution of spiral galaxies from late to early Hubble types (Friedli \& Martinet 1993; Hasan et al. 1993). The aim of this paper is to perform a systematic study of the photometric properties of bulges in earlytype barred galaxies. To date, this crucial piece of information is still missing. Few barred galaxies have been studied in such detail (e.g., Prieto et al. 1997, 2001) because of the complication introduced into the photometric analysis by the presence of 
the bar component. For example, there are claims that bars in early- and late-type disc galaxies have flat and exponential surface brightness profiles, respectively (Elmegreen et al. 1996), although the Sérsic profile (Sérsic 1968) has been adopted too (Peng et al. 2002).

By properly taking into account the bar contribution to the galaxy surface brightness we derive the structural parameters of SB0 bulges. To this purpose, we give a purely photometric and parametric definition of bulges following Fathi \& Peletier (2003). In particular, we assume that they are the inner photometric component of our sample galaxies, and that their surface brightness profile is described by the Sérsic law. The comparison with bulges in unbarred S0 and spiral galaxies will provide an insight as to whether SB0 bulges are barderived, disc-like, or otherwise different from classical earlytype bulges (Kormendy 1982, 1993; Kormendy \& Illingworth 1983; Möllenhoff et al. 1995; Seifert \& Scorza 1996; Erwin et al. 2003; Balcells et al. 2003).

This paper is organized as follows. In Sect. 2 we present an overview of the properties of the sample galaxies as well as their photometric observations and data analysis. In Sect. 3 we derive the photometric parameters of the structural components (bulge, disc, bar and lens) of the sample galaxies by applying a parametric photometric decomposition to their observed I-band surface brightness distribution. In Sect. 4 we compare the structural parameters of SB0 bars and bulges to those obtained in numerical simulations of early-type barred galaxies and to those derived for bulges of unbarred galaxies, respectively. Moreover, we derive the location of SB0 bulges on the fundamental plane and Faber-Jackson relation of elliptical galaxies and SO bulges by means of the central stellar velocity dispersion available for thirteen sample galaxies. In Sect. 5 we discuss a possible scenario for the formation of SB0 bulges.

\section{Sample selection, observations and basic data reduction}

We started a programme some years ago to enlarge the sample of early-type barred galaxies with pattern speeds measured using the Tremaine-Weinberg method (Tremaine \& Weinberg 1984), including galaxies of various bar strengths, luminosities, inclinations, etc. To this end, we selected a sample of fourteen SB0 objects by a visual inspection of their images in the Digitized Sky Survey. The galaxies were chosen for their brightness and undisturbed morphology, with no evidence of dust or spiral arms to complicate the Tremaine-Weinberg analysis, and a bar at an angle intermediate between the orientation of the major and minor axes. To date, we have derived the bar pattern speed of seven objects, namely NGC 1023 (Debattista et al. 2002), NGC 2950 (Corsini et al. 2003), ESO 139-G009, IC 874, NGC 1308, NGC 1440 and NGC 3412 (Aguerri et al. 2003). In this paper, we focus on the $I$-band photometric properties of the structural components of all the galaxies in the sample except for NGC 1023. Its high inclination (Debattista et al. 2002) prevents a reliable photometric decomposition from being obtained. In addition, we considered the SB0 galaxy NGC 7079, for which the bar pattern speed has been recently obtained by Debattista \& Williams (2004), who made their
I-band images available to us. A compilation of the main properties of the final collection of fourteen early-type barred galaxies is given in Table 1 .

The photometric observations of sample galaxies were carried out during several observing runs between 1997 and 2003 at the Cerro Tololo Inter-American Observatory (CTIO, Chile), at the European Southern Observatory (ESO) in La Silla (Chile), and at the Roque de los Muchachos Observatory (ORM) on La Palma (Spain). The log of observations and details of the different instrumental setups are given in Table 2 .

All images were reduced using standard IRAF ${ }^{1}$ tasks. We first subtracted a bias frame consisting of ten exposures for each night. The images were flat-fielded using sky flats taken at the beginning and/or end of each observing night. The sky background level was removed by fitting a second-order polynomial to the regions free of sources in the images. Special care was taken during sky subtraction to reach the outermost parts of the objects. Cosmic rays were removed by combining the different exposures using field stars as a reference and adopting a sigma clipping rejection algorithm. For the photometric calibration of the galaxies, standard stars of known magnitudes were observed. The calibration constant includes corrections for atmospheric and Galactic extinction, and a colour term. No attempt was made to correct for internal extinction. The atmospheric extinction was taken from the differential aerosol extinction for CTIO (Baldwin \& Stone 1984), ESO (Burki et al. 1995) and the ORM (King 1985). The Galactic extinction in the $B$ band was taken from Schlegel et al. (1998). We used the Galactic extinction law from Cardelli et al. (1989) in order to get the extinction in the $I$ band. Figure 1 shows the calibrated $I$-band images of the sample galaxies.

\section{Photometric parameters of the galaxy structural components}

\subsection{Decomposition of the radial surface brightness profiles}

We modeled the $I$-band surface brightness distribution of the sample galaxies as the sum of the contribution of their structural components (i.e., bulge, disc, bar and possibly a lens) by adopting the photometric decomposition technique developed by Prieto et al. (2001). The surface brightness distribution of each individual component has been assumed to follow a parametric law, which has to be strictly considered as an empirical fitting function.

For the radial surface brightness profile of the bulge we assumed the Sérsic law,

$I_{\text {bulge }}(r)=I_{\mathrm{e}} 10^{-b_{n}\left[\left(r / r_{\mathrm{e}}\right)^{1 / n}-1\right]}$,

where $r_{\mathrm{e}}, I_{\mathrm{e}}$ and $n$ are the effective (or half-light) radius, the surface brightness at $r_{\mathrm{e}}$, and a shape parameter describing the curvature of the profile, respectively. The value of $b_{n}$ is coupled to $n$ so that half of the total flux is always within $r_{\mathrm{e}}$ and can be approximated as $b_{n}=0.868 n-0.142$ (Caon et al. 1993).

1 IRAF is distributed by NOAO, which is operated by AURA Inc., under contract with the National Science Foundation. 
Table 1. Parameters of the sample galaxies.

\begin{tabular}{|c|c|c|c|c|c|c|c|c|c|c|}
\hline Galaxy & $\begin{array}{c}\text { Type } \\
\text { (RC3) } \\
(2)\end{array}$ & $\begin{array}{c}i \\
\left({ }^{\circ}\right) \\
(3)\end{array}$ & $\begin{array}{l}\text { PA } \\
\left({ }^{\circ}\right) \\
(4)\end{array}$ & $\begin{array}{c}B_{T} \\
(\mathrm{mag}) \\
(5)\end{array}$ & $\begin{array}{c}D_{25} \times d_{25} \\
(\operatorname{arcsec}) \\
(6)\end{array}$ & $\begin{array}{c}V_{\mathrm{CMB}} \\
\left(\mathrm{km} \mathrm{s}^{-1}\right) \\
(7)\end{array}$ & $\begin{array}{c}D \\
(\mathrm{Mpc}) \\
(8)\end{array}$ & $\begin{array}{c}M_{B_{T}}^{0} \\
(\mathrm{mag}) \\
(9)\end{array}$ & $\begin{array}{c}\sigma_{0} \\
\left(\mathrm{~km} \mathrm{~s}^{-1}\right) \\
(10)\end{array}$ & (11) \\
\hline ESO 139-G009 & (R)SAB0(rs) & 46 & 94 & 14.35 & $74 \times 60$ & 5389 & 71.9 & -20.28 & 196 & 1 \\
\hline IC 874 & SB0(rs) & 39 & 27 & 13.60 & $60 \times 43$ & 2602 & 34.7 & -19.41 & 99 & 1 \\
\hline IC 4796 & SB0(s): & 55 & 126 & 13.25 & $95 \times 56$ & 2983 & 39.8 & -20.13 & 143 & 2 \\
\hline NGC 357 & SB0(r): & 36 & 20 & 13.12 & $144 \times 104$ & 2136 & 28.5 & -19.78 & 178 & 2 \\
\hline NGC 364 & (R)SB0(s): & 46 & 27 & 15.61 & $85 \times 76$ & 4883 & 65.1 & -18.68 & ... & $\ldots$ \\
\hline NGC 936 & $\mathrm{SB} 0^{+}(\mathrm{rs})$ & 49 & 136 & 11.16 & $281 \times 244$ & 1100 & 14.7 & -19.85 & 204 & 2 \\
\hline NGC 1308 & SB0(r) & 36 & 59 & 14.70 & $70 \times 51$ & 6180 & 82.4 & -19.88 & 219 & 1 \\
\hline NGC 1440 & $\left(\mathrm{R}^{\prime}\right) \mathrm{SB} 0(\mathrm{rs}):$ & 38 & 26 & 12.90 & $128 \times 97$ & 1382 & 18.4 & -18.90 & 195 & 1 \\
\hline NGC 2950 & (R)SB0(r) & 46 & 116 & 11.82 & $161 \times 107$ & 1510 & 20.1 & -19.79 & 164 & 3 \\
\hline NGC 3412 & $\mathrm{SB} 0(\mathrm{~s})$ & 55 & 151 & 11.43 & $218 \times 123$ & 1202 & 16.0 & -19.73 & 112 & 1 \\
\hline NGC 3941 & $\mathrm{SB} 0(\mathrm{~s})$ & 49 & 9 & 11.23 & $208 \times 137$ & 1183 & 15.8 & -19.87 & 137 & 4 \\
\hline NGC 4340 & $\mathrm{SB}^{+}(\mathrm{r})$ & 49 & 95 & 12.11 & $213 \times 169$ & 1238 & 16.5 & -19.11 & 118 & 5 \\
\hline NGC 6684 & $\left(\mathrm{R}^{\prime}\right) \mathrm{SB} 0(\mathrm{~s})$ & 49 & 36 & 11.29 & $239 \times 158$ & 837 & 11.2 & -19.26 & 112 & 2 \\
\hline NGC 7079 & $\mathrm{SB} 0(\mathrm{~s})$ & 52 & 82 & 12.49 & $128 \times 79$ & 2541 & 33.9 & -20.34 & 165 & 2 \\
\hline
\end{tabular}

NOTE. Column (2): morphological classification from de Vaucouleurs et al. (1991, hereafter RC3); Col. (3): inclination of the galaxy disc from this paper; Col. (4): major-axis position angle of the galaxy disc from this paper; Col. (5): total observed blue magnitude from from Lyon Extragalactic Database (hereafter LEDA); Col. (6): apparent isophotal diameters measured at a surface-brightness level of $\mu_{B}=25 \mathrm{mag} \mathrm{arcsec}^{-2}$ from RC3; Col. (7): radial velocity with respect to the CMB radiation from LEDA; Col. (8): distance obtained as $V_{\mathrm{CMB}} / H_{0}$ with $H_{0}=75 \mathrm{~km} \mathrm{~s}^{-1} \mathrm{Mpc}^{-1}$; Col. (9): absolute total blue magnitude from $B_{T}$ corrected for inclination and extinction as in LEDA and adopting $D$; Col. (10): central velocity dispersion of the stellar component after correcting to the equivalent of an aperture of radius $r_{\mathrm{e}} / 8$ following the prescription by Jorgensen et al. (1995), with $r_{\mathrm{e}}$ the effective radius of the bulge from Table 3 . Typical error is $10 \mathrm{~km} \mathrm{~s}{ }^{-1}$; Col. (11): list of references for the central velocity dispersion: 1 = Aguerri et al. (2003), 2 = Wegner et al. (2003), 3 = Corsini et al. (2003), $4=$ Fisher (1997), $5=$ Prugniel \& Simien (1997).

For the radial surface brightness profile of the disc we assumed the exponential law (Freeman 1970)

$I_{\text {disc }}(r)=I_{0} \mathrm{e}^{-r / h}$,

where $h$ and $I_{0}$ are the scale length and central surface brightness of the disc, respectively.

For the radial surface brightness profile of the bar we assumed either a flat profile (Prieto et al. 1997),

$I_{\mathrm{bar}}(r)=I_{\mathrm{b}} /\left(1+\mathrm{e}^{\frac{r-r_{\mathrm{b}}}{r_{\mathrm{s}}}}\right)$

or a Freeman bar profile (Freeman 1966),

$I_{\mathrm{bar}}(r)=I_{\mathrm{b}} \sqrt{1-\left(\frac{r}{r_{\mathrm{b}}}\right)^{2}}$,

where $r_{\mathrm{b}}, r_{\mathrm{s}}$ and $I_{\mathrm{b}}$ are the bar length, a scale length, and the bar central surface brightness, respectively.

For the radial surface brightness profile of the lens we assumed the law by Duval \& Athanassoula (1983),

$I_{\text {lens }}(r)=I_{1}\left(1-\left(\frac{r}{r_{1}}\right)^{2}\right)$,

where $r_{1}$ and $I_{1}$ are the length and central surface brightness of the lens.
Following Prieto et al. (2001), we extracted the radial surface brightness profiles for each sample object along the major axis of the bar from the deprojected image of each galaxy. Image deprojection was performed by means of a fluxconserving stretch of the observed image along the disc minor axis, and adopting the disc inclination and position angle given in Table 1 . They were measured by averaging the values in the outermost region of the ellipticity and position angle radial profiles, which were obtained by fitting elliptical isophotes with the ELLIPSE task in IRAF. In all cases, we first masked foreground stars and bad pixels and then we fitted ellipses allowing their centres to vary. Within the errors, no variation in the ellipse centres was found for the galaxies studied in this paper. The final ellipse fits were done at fixed ellipse centres. The inclination and position angle of the galaxies were determined by averaging the outer isophotes, and their typical errors are $\lesssim 1^{\circ}$.

Iterative fitting of the model surface brightness $I_{\text {mod }}=$ $I_{\text {bulge }}+I_{\text {disc }}+I_{\text {bar }}+I_{\text {lens }}$ to the extracted radial surface brightness profiles resulted in a non-linear system of equations for the free parameters. These ranged from 7 (when bulge, disc and Freeman bar were fitted) to 12 (when bulge, disc, flat bar and lens were fitted). A Levenberg-Marquardt algorithm (Press et al. 1992) was used for the solution of this system. We first fitted the radial surface brightness profiles by assuming the bulge shape parameter to be $n=0.5,1,1.5, \ldots, 5$. The result corresponding to the lowest value of $\chi^{2}$ yielded a rough guess 

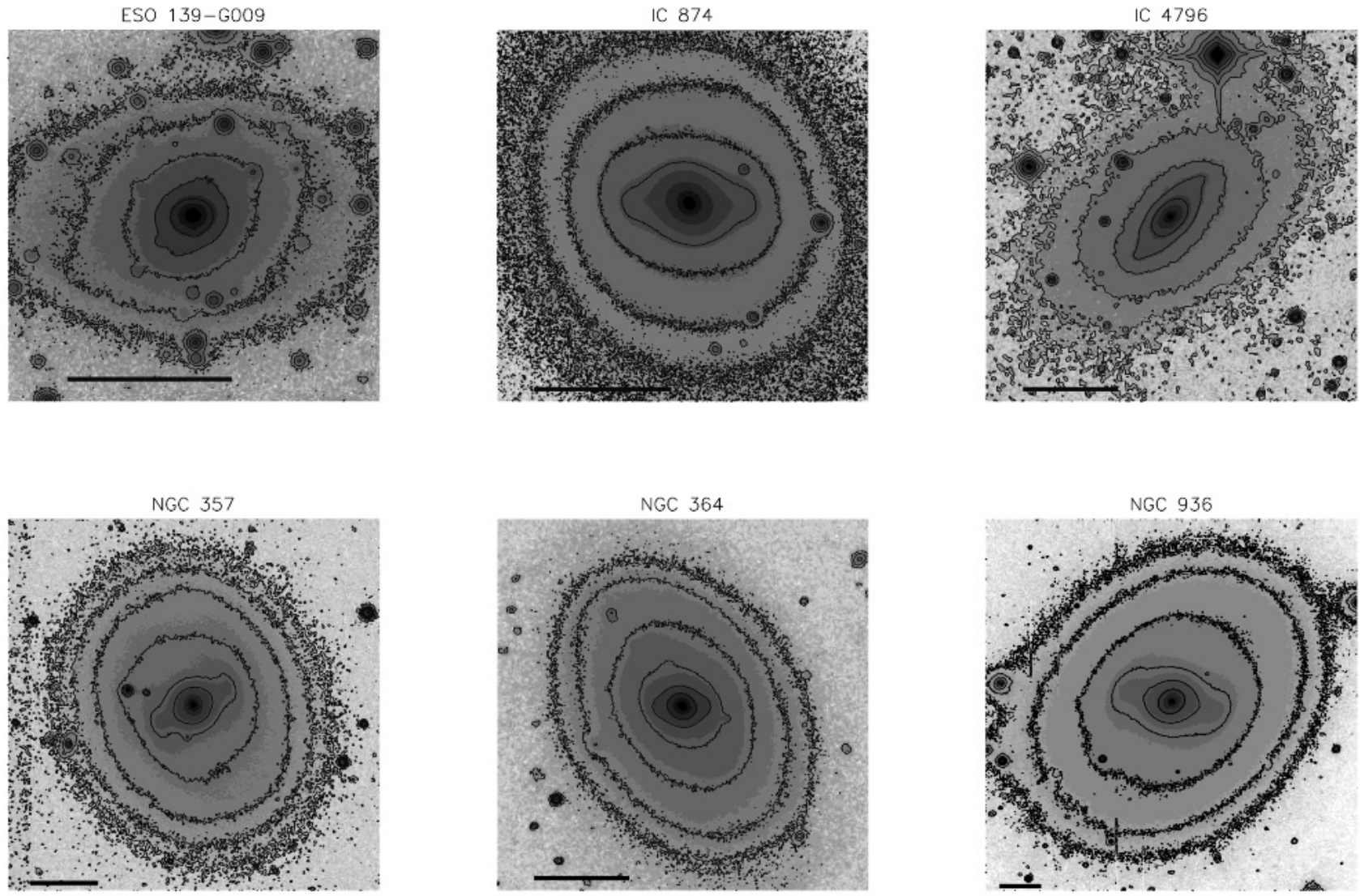

NGC 1308

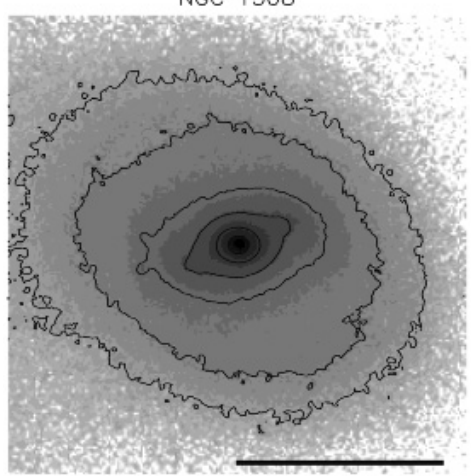

NGC 1440

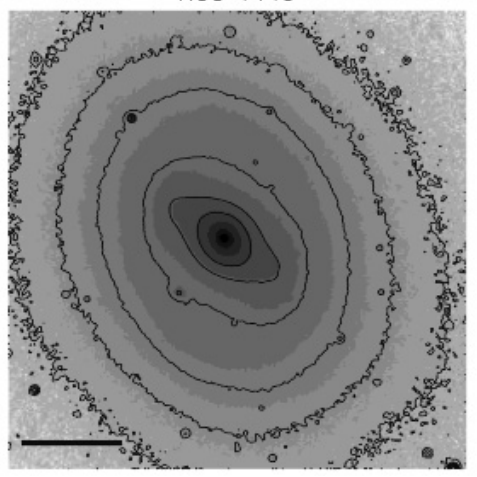

NGC 2950

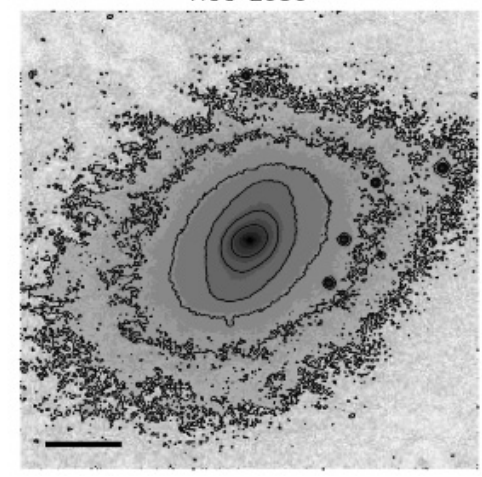

Fig. 1. I-band images of the galaxies: IC 4796, NGC 357, NGC 364, NGC 936, NGC 2950, NGC 3941, NGC 4340, NGC 6684 and NGC 7079. In each panel north is at the top and east is to the left, and the bottom horizontal line is 30 arcsec in length. The isocontours are spaced at $1.0 \mathrm{mag} \operatorname{arcsec}^{-2}$, with the outermost contour corresponding to the surface brightness at Col. 9 of Table 2.

for the initial values of the parameters in the LevenbergMarquardt iteration, where all the parameters, including $n$, were allowed to vary. This approach ensures a robust estimate of $n$ and is similar to the one extensively tested by MacArthur et al. (2003).

The fit of the observed surface brightness profiles was done to intensities. Errors in intensities were used in the fit. For all the sample galaxies the best fit of the observed surface brightness was obtained with a flat bar, except for NGC 3412 and NGC 6684, for which a Freeman bar was adopted. A lens component was included to account for the light bump observed at large radii in the radial surface brightness profiles of ESO 139-G009, IC 874, NGC 364, NGC 936, NGC 1440, NGC 3412 and NGC 7079. Although it decreases the fit residuals, the choice of such a lens component is somewhat arbitrary and alternative solutions like a ring (e.g., Duval \& Athanassoula 1983) or a truncated disk (e.g., Pohlen et al. 2002) have not been considered. For this reason, we will discuss how the photometric parameters of the remaining structural components and their relations are affected by the presence of the lens component. On the other hand, we did not consider as further components the secondary stellar bars hosted by NGC 357, NGC 2950, NGC 3941, NGC 4340, and NGC 6684. These secondary bars have a small size $\left(\simeq 6^{\prime \prime}\right)$ and are not 

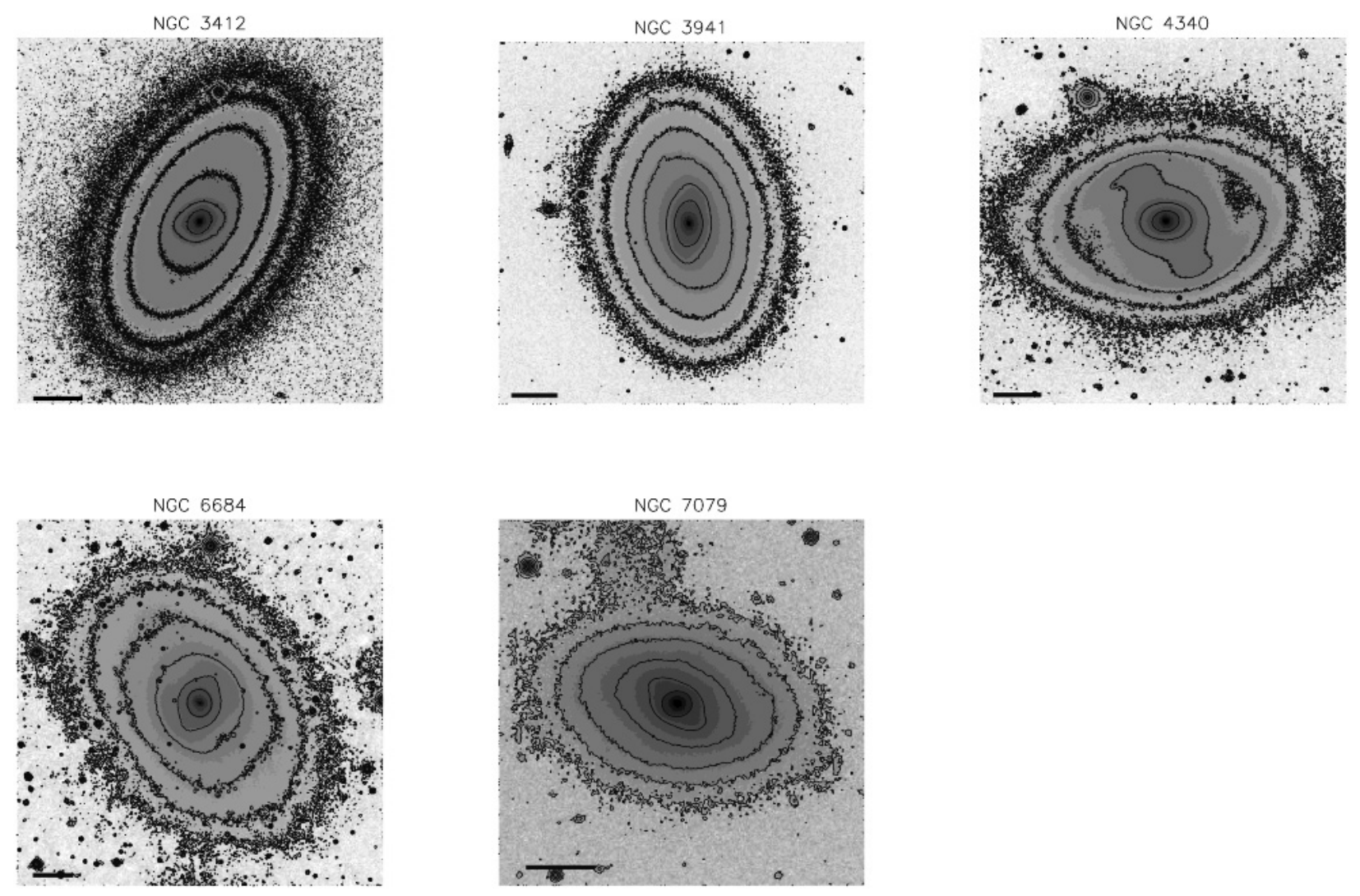

Fig. 1. continued.

aligned with the major axis of the main bar (Erwin 2004, and references therein). This means that their contribution to the surface brightness profile observed along the main bar major axis is negligible and does not affect the bulge parameters we obtained from such profiles.

The parameters derived for the structural components of the sample galaxies are collected in Table 3 . The formal errors of the fit are given in Table 3 too. They are most probably underestimated since they do not include the other uncertainties, like those in the sky background determination. The result of the photometric decomposition of the radial surface brightness profiles extracted along the bar major axis of the sample galaxies is shown in Fig. 2.

\subsection{Seeing effects}

The ground-based images are affected by seeing, which scatters the light of the objects and produces a loss of spatial resolution. This is particularly critical in the central regions of galaxies, where the slope of the radial surface brightness profile is steeper. Since the bulge contribution dominates the surface brightness distribution at small radii, seeing mostly affects bulge structural parameters. Seeing effects on the scale parameters of a Sérsic surface brightness profile have been extensively discussed by Trujillo et al. (2001a,b).

To deal with this limitation, we fitted the observed radial surface brightness profiles at radii larger than twice the $F W H M$ of the point spread function (PSF). The PSF was approximated with a circular two-dimensional Gaussian and its $F W H M$ was derived by fitting several field stars in the final combined images of the sample galaxies (Table 2). To estimate systematic errors due to the seeing on the fitted parameters of the bulge, we applied the adopted parametric decomposition technique to a set of artificial disc galaxies. We generated 250 images of galaxies with a Sersic bulge and an exponential disc with surface brightnesses and scale lengths randomly chosen in the range of the values that we observed for the sample galaxies (Table 3 ). This has been done by satisfying all the following conditions: $2^{\prime \prime} \leq r_{\mathrm{e}} \leq 10^{\prime \prime}, 0.8 \leq n \leq 3,12^{\prime \prime} \leq h \leq 40^{\prime \prime}$, $0 \leq B / D \leq 0.5$, and $11 \leq m_{I} \leq 14$.

The simulated images have been built to have the pixel scale, gain and readout noise of the worst-resolved images of our dataset, namely those obtained with the $1.54 \mathrm{~m}$ Danish telescope. An appropriate level of noise was added to yield a signal-to-noise ratio similar to that of the images we obtained during our observing runs. Finally, all the artificial images have been convolved with a circular two-dimensional Gaussian of $F W H M=1{ }^{\prime \prime} 0$ which is the typical seeing $F W H M$ of our data (Table 2).

The images of the artificial galaxies have been analyzed as if they were real. The fitting algorithm recovered slightly fainter scale surface brightnesses, larger scale lengths and larger bulge shape parameters with respect to the input values. The mean relative errors on the fitted parameters are $\left\langle\Delta \mu_{\mathrm{e}}\right\rangle=-0.01,\left\langle\Delta r_{\mathrm{e}}\right\rangle=0.02$, and $\langle\Delta n\rangle=0.02$ for the bulge, $\left\langle\Delta \mu_{0}\right\rangle \lesssim-0.01$ and $\langle h\rangle=0.01$ for the disc, providing the reliability and accuracy of the bulge parameters derived. We suspect that seeing affects bar parameters in the same way as it does 

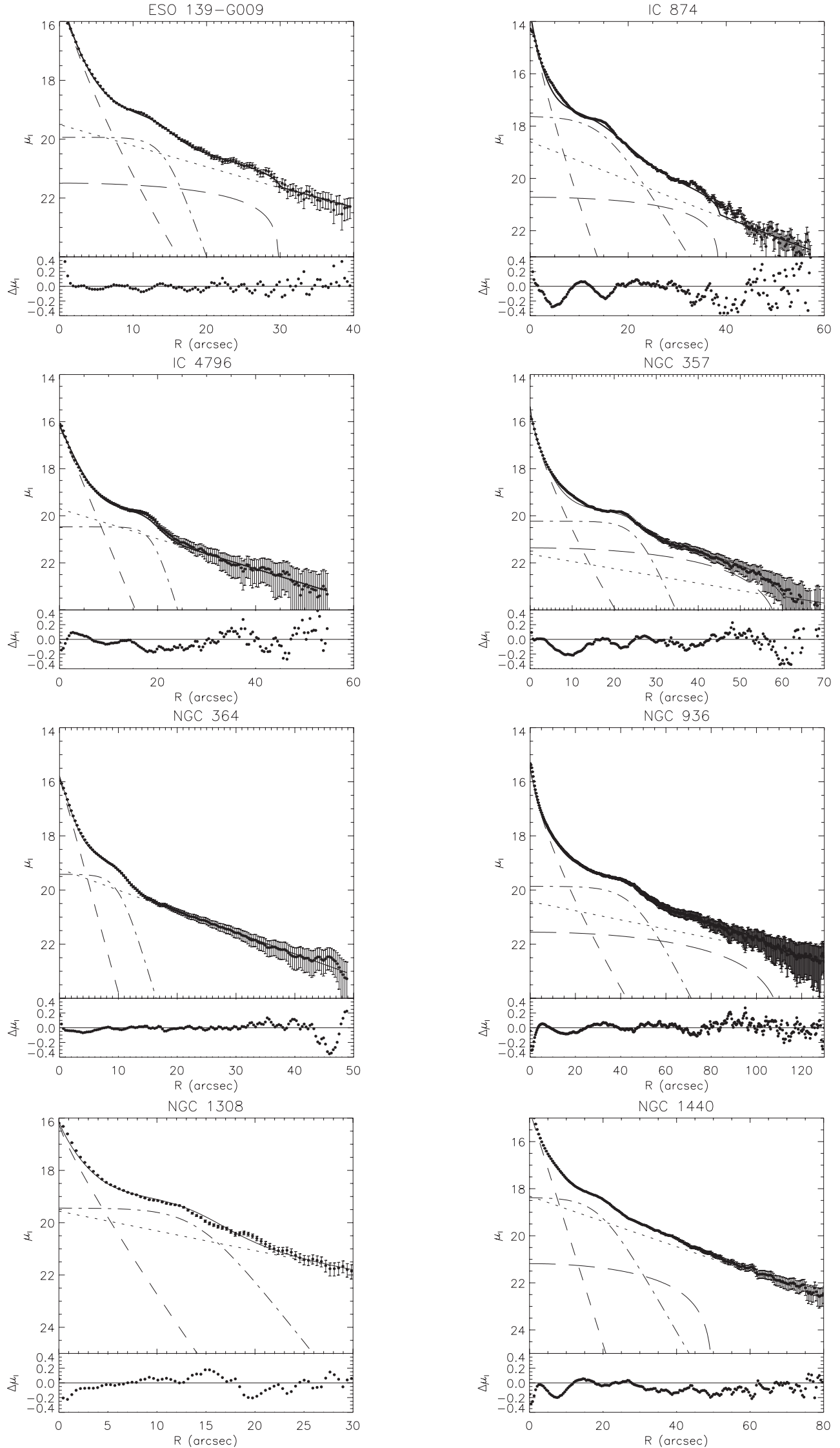

Fig. 2. Decomposition of the radial surface brightness profiles in the $I$-band along the major axis of the bar of the sample galaxies, and residuals of the fit. The adopted structural components are bulges (dashed line), discs (dotted line), bar (dash-dotted line) and lenses (long-dashed line). The continuous line represents the total model. 

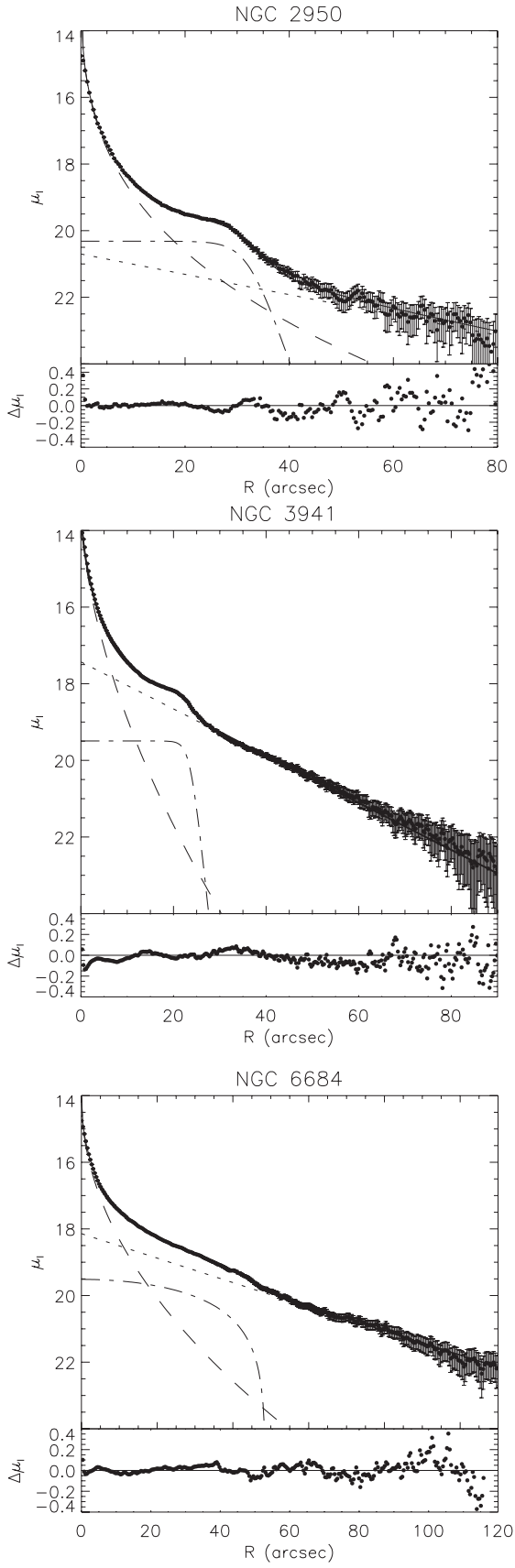

Fig. 2. continued.

disc parameters. In fact, the radial surface brightness profile of bars does not steepen toward the centre (Fig. 2) and the central surface brightnesses and scale lengths of bars are similar to those of discs (Table 3 ).

\subsection{Dependence of the result on the fitting model}

The reason for including a bar and possibly a lens in fitting the radial surface brightness profiles of SB0 galaxies is to reduce the large and systematic residuals obtained when only the bulge and disc are considered.

In order to investigate how the different structural parameters depend on the fitting procedure, we have performed two other fits: a photometric decomposition with only the bulge and
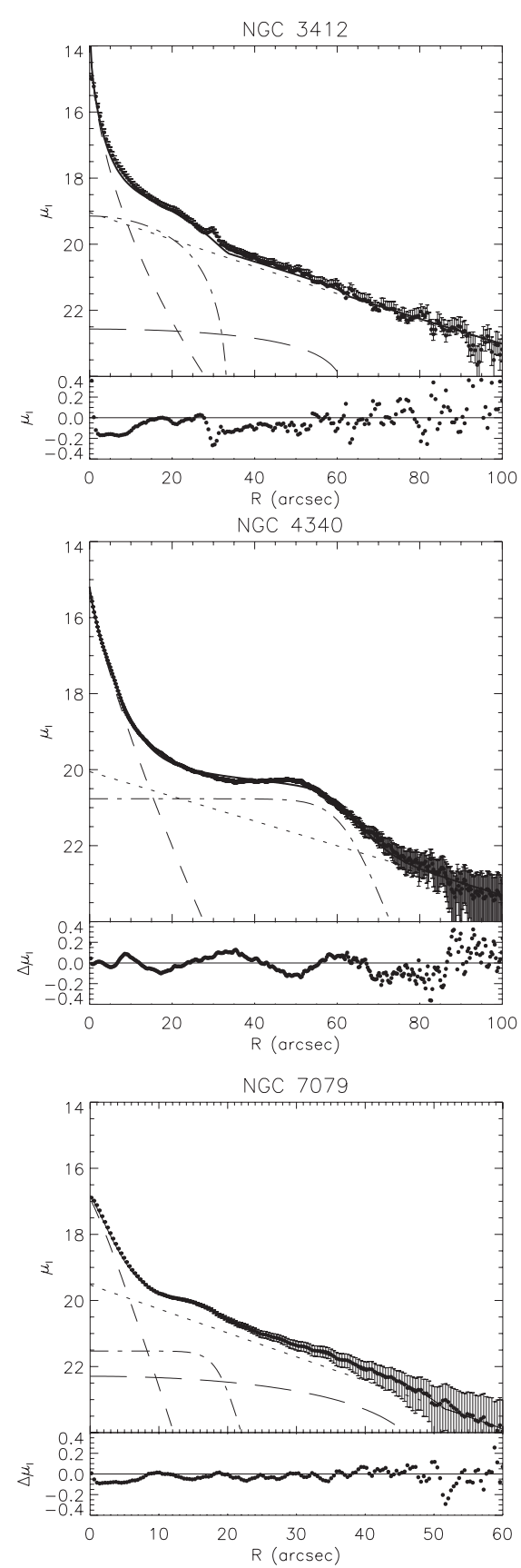

disc components, and a photometric decomposition with the bulge, disc and bar, but without the lens component. Table 4 lists the relative differences for the bulge and disc parameters if only bulge and disc structures are used in fitting the surface brightness profiles of the galaxies. In general, discs turn out to be smaller and brighter than those obtained from fits that include the bar and lens components. It should be noticed that the sample galaxies host a strong bar, which is not a negligible light component in the fitting procedure. Table 5 lists the relative differences of the bulge, disc and bar parameters if the lens is ignored in the fitting procedure. It turns out that ignoring the light contribution of the lens does not affect the bar parameters since these are characterized by negligible mean relative differences $\left(\left\langle\Delta \mu_{\mathrm{b}}\right\rangle=0.04,\left\langle\Delta r_{\mathrm{b}}\right\rangle=0.04\right)$. This is in disagreement 
Table 2. Log of the observations.

\begin{tabular}{|c|c|c|c|c|c|c|c|c|}
\hline Galaxy & Instrument & (3) & Pixel \# & $\begin{array}{c}\text { Scale } \\
(" / \text { pixel }) \\
(5)\end{array}$ & (6) & $\begin{array}{c}\text { Exp. time } \\
\text { (s) } \\
(7)\end{array}$ & $\begin{array}{l}\text { Seeing } \\
\left({ }^{\prime \prime}\right) \\
(8)\end{array}$ & $\begin{array}{c}\mu_{I} \\
\left(\mathrm{mag} / \operatorname{arcsec}^{2}\right) \\
(9) \\
\end{array}$ \\
\hline ESO 139-G009 & NTT+EMMI & TK2048 EB & $2048 \times 4096$ & 0.270 & 2001 May 23 & $300^{g}$ & 1.2 & 22.05 \\
\hline IC 874 & NTT+EMMI & TK2048 EB & $2048 \times 4096$ & 0.270 & 2001 May 23 & $300^{g}$ & 0.9 & 23.00 \\
\hline IC 4796 & Danish+DFOSC & MAT/EEV & $2048 \times 4096$ & 0.390 & 2002 May 08 & $1260^{g}$ & 1.1 & 23.33 \\
\hline NGC 357 & NTT+EMMI & MIT/LL & $2 \times 2048 \times 4096$ & 0.332 & 2002 Oct. 05 & $300^{g}$ & 1.0 & 24.06 \\
\hline NGC 364 & Danish+DFOSC & MAT/EEV & $2048 \times 4096$ & 0.390 & 2001 Nov. 11 & $600^{g}$ & 1.2 & 23.25 \\
\hline NGC 936 & JKT & SITe2 & $2048 \times 2048$ & 0.330 & 2002 Sep. 09 & $1800^{h}$ & 1.5 & 22.80 \\
\hline NGC 1308 & JKT & SITe2 & $2048 \times 2048$ & 0.330 & 2001 Oct. 11 & $7200^{h}$ & 0.9 & 22.20 \\
\hline NGC 1440 & Danish+DFOSC & MAT/EEV & $2048 \times 4096$ & 0.390 & 2001 Nov. 11 & $3600^{g}$ & 1.1 & 22.53 \\
\hline NGC 2950 & JKT & SITe2 & $2048 \times 2048$ & 0.330 & 2000 Dec. 28 & $2700^{h}$ & 1.0 & 23.15 \\
\hline NGC 3412 & JKT & SITe2 & $2048 \times 2048$ & 0.330 & 2000 May 28 & $3600^{h}$ & 1.0 & 23.22 \\
\hline NGC 3941 & TNG+DOLORES & Loral & $2048 \times 2048$ & 0.275 & 2003 Mar. 11 & $120^{J}$ & 1.1 & 23.00 \\
\hline NGC 4340 & TNG+DOLORES & Loral & $2048 \times 2048$ & 0.275 & 2003 Mar. 11 & $180^{j}$ & 1.0 & 23.60 \\
\hline NGC 6684 & NTT+EMMI & MIT/LL & $2 \times 2048 \times 4096$ & 0.332 & 2002 Oct. 05 & $75^{g}$ & 1.2 & 22.28 \\
\hline NGC 7079 & CTIO 0.9-m & Tek 2K\#3 & $2048 \times 2046$ & 0.396 & 1997 Aug. 02 & $900^{g}$ & 1.5 & 23.80 \\
\hline
\end{tabular}

NOTE. Column (2): telescope and camera, NTT+EMMI = New Technology Telescope mounting the ESO Multi-Mode Instrument in Red Imaging and Low-Dispersion Spectroscopic Mode, Danish+DFOSC = $1.54 \mathrm{~m}$ Danish telescope mounting the Danish Faint Object Spectrograph and Camera, JKT $=$ Jacobus Kapteyn Telescope, TNG+DOLORES $=$ Telescopio Nazionale Galileo mounting the Device Optimized for the Low Resolution, CTIO 0.9-m = $0.9 \mathrm{~m}$ telescope at CTIO; Col. (7): total exposure time obtained with with Gunn $\left({ }^{g}\right)$, Harris $\left({ }^{h}\right)$ or Johnson $\left({ }^{j}\right)$ I-band filter; Col. (8): seeing FWHM measured by fitting a circular two-dimensional Gaussian to several field stars in the final combined image of the galaxy; Col. (9): surface brightness level at the outermost radius along the bar major axis.

with the claims by Valenzuela \& Klypin (2003), who argue that the bar length of the galaxies resulting from their numerical simulations could hardly be compared to that of the galaxies observed by Aguerri et al. (2003) because of the presence of a lens component.

\section{Parameter relations}

\subsection{Length and strength of the bar}

Measuring the bar length is not trivial task and several methods have been developed for this purpose (see Athanassoula \& Misiriotis 2002, for a review).

After measuring the bar length by means of the photometric decomposition $\left(r_{\mathrm{b}, 1}\right.$ in Table 6$)$, we derived it by means of Fourier decomposition of the galaxy surface brightness with the methods of Aguerri et al. (2000) and Debattista \& Sellwood (2000).

The method of Aguerri et al. (2000) is based on the ratios of the intensities in the bar and the inter-bar region. The azimuthal surface brightness profile of the deprojected galaxies is decomposed into a Fourier series. The bar intensity, $I_{\mathrm{b}}$, then is defined as $I_{\mathrm{b}}=I_{0}+I_{2}+I_{4}+I_{6}$ (where $I_{0}, I_{2}, I_{4}$ and $I_{6}$ are the $m=0,2$, 4 and 6 terms of the Fourier decomposition, respectively). The inter-bar intensity is defined as $I_{\mathrm{ib}}=I_{0}-I_{2}+I_{4}-I_{6}$. The bar region is defined as the region where

$$
I_{\mathrm{b}} / I_{\mathrm{ib}}>0.5\left[\max \left(I_{\mathrm{b}} / I_{\mathrm{ib}}\right)-\min \left(I_{\mathrm{b}} / I_{\mathrm{ib}}\right)\right]+\min \left(I_{\mathrm{b}} / I_{\mathrm{ib}}\right)
$$

Thus the semi-major axis of the bar is identified as the outer radius at which

$I_{\mathrm{b}} / I_{\mathrm{ib}}=0.5\left[\max \left(I_{\mathrm{b}} / I_{\mathrm{ib}}\right)-\min \left(I_{\mathrm{b}} / I_{\mathrm{ib}}\right)\right]+\min \left(I_{\mathrm{b}} / I_{\mathrm{ib}}\right)$.

This is given as $r_{\mathrm{b}, 2}$ in Table 6 for all the sample galaxies. According to the results by Athanassoula \& Misiriotis (2002), based on numerical simulations, the error in bar length obtained by the analysis of the Fourier amplitudes ranges between $4 \%$ and $8 \%$.

The method of Debattista \& Sellwood (2000) is also based on a Fourier decomposition of the surface brightness distribution. It assumes that the bar can extend only in the radial interval where the phase of the $m=2$ moment is constant to within the errors. Our sample galaxies were selected to be free of spirals and rings to prevent the overestimation of the bar length which is listed as $r_{\mathrm{b}, 3}$ in Table 6.

A related, but not equivalent, method uses the phases of the ellipse fits (Debattista \& Williams 2004), which then gives our fourth estimate of the bar length $r_{\mathrm{b}, 4}$ in Table 6 . The ellipses fitting the galaxy isophotes are deprojected and their phase angle is measured. The bar length is then the largest radius out to which the phases are consistent with a constant, taking into account that deprojecting the bulge, which we did not subtract from our image for this analysis, results in a twist interior to the bar.

The mean of $r_{\mathrm{b}, 1}, r_{\mathrm{b}, 2}, r_{\mathrm{b}, 3}$ and $r_{\mathrm{b}, 4}$ is our best estimate of the bar length $r_{\mathrm{b}}$ and we used the largest deviations from the mean for the error estimates (Table 6). 
Table 3. Photometric parameters of the sample galaxies.

\begin{tabular}{|c|c|c|c|c|c|c|c|c|c|c|}
\hline Galaxy & $\begin{array}{c}\mu_{\mathrm{e}} \\
\left(\mathrm{mag} / \operatorname{arcsec}^{2}\right) \\
(2)\end{array}$ & $\begin{array}{c}r_{\mathrm{e}} \\
(\operatorname{arcsec}) \\
(3)\end{array}$ & (4) & $\begin{array}{c}\mu_{0} \\
\left(\mathrm{mag} / \operatorname{arcsec}^{2}\right) \\
(5)\end{array}$ & $\begin{array}{c}h \\
(\operatorname{arcsec}) \\
(6)\end{array}$ & $\begin{array}{c}\mu_{\mathrm{b}} \\
\left(\mathrm{mag} / \operatorname{arcsec}^{2}\right) \\
(7)\end{array}$ & $\begin{array}{c}r_{\mathrm{b}} \\
(\operatorname{arcsec}) \\
(8)\end{array}$ & $\begin{array}{c}r_{\mathrm{s}} \\
(\operatorname{arcsec}) \\
(9)\end{array}$ & $\begin{array}{c}\mu_{\mathrm{l}} \\
\left(\mathrm{mag} / \operatorname{arcsec}^{2}\right) \\
(10)\end{array}$ & $\begin{array}{c}r_{1} \\
(\operatorname{arcsec}) \\
(11)\end{array}$ \\
\hline ESO 139-G009 & $17.30 \pm 0.05$ & $2.86 \pm 0.07$ & $1.35 \pm 0.03$ & $19.51 \pm 0.15$ & $15.12 \pm 1.08$ & $19.94 \pm 0.07$ & $14.40 \pm 0.10$ & $1.51 \pm 0.10$ & $21.49 \pm 0.11$ & $30.34 \pm 0.16$ \\
\hline IC 874 & $15.97 \pm 0.12$ & $2.40 \pm 0.15$ & $1.28 \pm 0.05$ & $18.59 \pm 0.08$ & $14.70 \pm 0.90$ & $17.62 \pm 0.02$ & $15.00 \pm 0.04$ & $3.53 \pm 0.05$ & $20.72 \pm 0.02$ & $38.61 \pm 0.04$ \\
\hline IC 4796 & $18.02 \pm 0.03$ & $3.61 \pm 0.02$ & $1.00 \pm 0.32$ & $19.70 \pm 0.04$ & $16.94 \pm 0.15$ & $20.47 \pm 0.02$ & $19.11 \pm 0.08$ & $1.56 \pm 0.07$ & & \\
\hline NGC 357 & $18.05 \pm 0.03$ & $3.93 \pm 0.03$ & $1.40 \pm 0.08$ & $21.63 \pm 0.21$ & $34.86 \pm 3.80$ & $20.23 \pm 0.07$ & $25.41 \pm 0.08$ & $1.64 \pm 0.05$ & $21.36 \pm 0.06$ & $60.06 \pm 1.37$ \\
\hline NGC 364 & $17.66 \pm 0.02$ & $2.39 \pm 0.01$ & $0.99 \pm 0.03$ & $19.22 \pm 0.02$ & $13.84 \pm 0.07$ & $19.41 \pm 0.02$ & $10.21 \pm 0.03$ & $1.48 \pm 0.02$ & & \\
\hline NGC 936 & $18.08 \pm 0.04$ & $8.36 \pm 0.18$ & $1.40 \pm 0.12$ & $20.42 \pm 0.28$ & $63.72 \pm 6.70$ & $19.86 \pm 0.05$ & $46.20 \pm 1.05$ & $6.62 \pm 0.05$ & $21.55 \pm 0.08$ & $113.85 \pm 2.57$ \\
\hline NGC 1308 & $18.05 \pm 0.05$ & $2.31 \pm 0.03$ & $1.15 \pm 0.23$ & $19.56 \pm 0.07$ & $14.39 \pm 0.07$ & $19.44 \pm 0.05$ & $14.17 \pm 1.32$ & $2.51 \pm 0.07$ & & \\
\hline NGC 1440 & $16.48 \pm 0.02$ & $3.65 \pm 0.08$ & $1.03 \pm 0.07$ & $18.31 \pm 0.13$ & $20.25 \pm 0.89$ & $18.37 \pm 0.02$ & $19.23 \pm 0.05$ & $4.13 \pm 0.05$ & $21.17 \pm 0.07$ & $50.35 \pm 0.32$ \\
\hline NGC 2950 & $18.48 \pm 0.07$ & $8.16 \pm 0.10$ & $3.06 \pm 0.53$ & $20.70 \pm 0.12$ & $35.61 \pm 0.95$ & $20.31 \pm 0.06$ & $32.20 \pm 0.83$ & $2.38 \pm 0.03$ & & \\
\hline NGC 3412 & $17.21 \pm 0.03$ & $3.83 \pm 0.06$ & $2.00 \pm 0.30$ & $18.98 \pm 0.13$ & $26.42 \pm 1.16$ & $19.13 \pm 0.03$ & $34.00 \pm 2.32$ & & $22.24 \pm 0.04$ & $63.00 \pm 2.18$ \\
\hline NGC 3941 & $16.72 \pm 0.02$ & $4.24 \pm 0.02$ & $1.70 \pm 0.01$ & $17.43 \pm 0.02$ & $17.70 \pm 0.06$ & $19.49 \pm 0.03$ & $23.63 \pm 0.03$ & $0.96 \pm 0.03$ & & \\
\hline NGC 4340 & $17.60 \pm 0.03$ & $5.32 \pm 0.01$ & $1.27 \pm 0.01$ & $20.05 \pm 0.02$ & $33.38 \pm 0.15$ & $20.76 \pm 0.04$ & $61.51 \pm 0.06$ & $3.88 \pm 0.05$ & & \\
\hline NGC 6684 & $18.05 \pm 0.08$ & $8.77 \pm 0.20$ & $2.00 \pm 0.13$ & $18.14 \pm 0.05$ & $29.56 \pm 1.35$ & $19.58 \pm 0.07$ & $48.75 \pm 1.51$ & & & \\
\hline NGC 7079 & $18.51 \pm 0.01$ & $3.20 \pm 0.01$ & $0.90 \pm 0.04$ & $19.53 \pm 0.41$ & $14.96 \pm 1.72$ & $21.53 \pm 0.10$ & $18.95 \pm 0.08$ & $1.40 \pm 0.07$ & $22.29 \pm 0.15$ & $51.48 \pm 0.57$ \\
\hline
\end{tabular}

NOTE. Column (2): effective surface brightness of bulge; Col. (3) effective radius of bulge; Col. (4): shape parameter of bulge; Col. (5): central surface brightness of disc; Col. (6): scale length of disc; Col. (7): central surface brightness of bar; Col. (8): bar length; Col. (9): scale length of flat bar; Col. (10): central surface brightness of lens; Col. (6): length of lens. 
Table 4. Relative differences in the structural parameters if only bulge and disc components are fitted to the surface brightness profiles of the galaxies. They are defined as $\Delta X=\frac{X_{0}-X_{1}}{X_{0}}$ where $X_{0}$ is the parameter when all components are fitted and $X_{1}$ is the parameter when only bulge and disc components are used.

\begin{tabular}{lrrrrc}
\hline \hline \multicolumn{1}{c}{ Galaxy } & \multicolumn{1}{c}{$\Delta \mu_{\mathrm{e}}$} & \multicolumn{1}{c}{$\Delta r_{\mathrm{e}}$} & \multicolumn{1}{c}{$\Delta n$} & \multicolumn{1}{c}{$\Delta \mu_{0}$} & \multicolumn{1}{c}{$\Delta h$} \\
\multicolumn{1}{c}{$(1)$} & \multicolumn{1}{c}{$(2)$} & \multicolumn{1}{c}{$(3)$} & \multicolumn{1}{c}{$(4)$} & \multicolumn{1}{c}{$(5)$} & \multicolumn{1}{c}{$(6)$} \\
\hline ESO 139-G009 & -0.01 & 0.41 & 0.27 & 0.08 & 0.58 \\
IC 874 & -0.04 & 0.01 & -0.18 & 0.10 & 0.37 \\
IC 4796 & -0.01 & 0.21 & -0.09 & 0.06 & 0.37 \\
NGC 357 & -0.01 & 0.18 & 0.09 & 0.14 & 0.58 \\
NGC 364 & -0.03 & -0.38 & -0.40 & 0.03 & 0.26 \\
NGC 936 & -0.01 & 0.13 & -0.23 & 0.01 & 0.52 \\
NGC 1308 & 0.01 & 0.44 & 0.15 & 0.09 & 0.59 \\
NGC 1440 & -0.05 & -0.59 & -1.06 & 0.05 & 0.26 \\
NGC 2950 & 0.06 & 0.60 & 0.34 & 0.12 & 0.64 \\
NGC 3412 & 0.01 & 0.31 & 0.01 & 0.06 & 0.55 \\
NGC 3941 & 0.02 & 0.24 & 0.13 & 0.05 & 0.43 \\
NGC 4340 & 0.01 & 0.18 & 0.12 & 0.07 & 0.31 \\
NGC 6684 & 0.03 & 0.08 & 0.10 & 0.09 & 0.20 \\
NGC 7079 & -0.18 & 0.33 & 0.17 & 0.05 & 0.47 \\
\hline
\end{tabular}

The bar strength $s_{\mathrm{b}}$ of each sample galaxies is also listed Table 6 and is defined as

$$
s_{\mathrm{b}}=\frac{1}{r_{\mathrm{b}, 2}-r_{\mathrm{in}}} \int_{r_{\mathrm{in}}}^{r_{\mathrm{b}, 2}} \frac{I_{2}}{I_{0}}(r) \mathrm{d} r,
$$

where $r_{\text {in }}$ and $r_{\mathrm{b}, 2}$ are the inner and outer radius of the bar region defined by Eq. (6), while $I_{0}$ and $I_{2}$ are the $m=0$ and $m=2$ terms of the Fourier decomposition, respectively. Although ESO 139-G009 is classified as a weakly barred S0 (RC3), its bar strength is comparable to that of the other SB0 galaxies in the sample.

We found a mean bar-to-disc scale length ratio $\left\langle r_{\mathrm{b}} / h\right\rangle=$ $1.21 \pm 0.08$. This becomes $\left\langle r_{\mathrm{b}} / h\right\rangle=1.42 \pm 0.09$ if lenses are excluded from the surface brightness fit. This is in agreement with the findings of the recent numerical simulations of barred galaxies by: O'Neil \& Dubinski $\left(2003,\left\langle r_{\mathrm{b}} / h\right\rangle=1.1 \pm 0.33\right)$, Valenzuela \& Klypin $\left(2003,<r_{\mathrm{b}} / h>=1.0-1.2\right)$.

\subsection{Bulge and disc interplay}

From NIR observations of spiral galaxies, Andredakis et al. (1995) found a correlation between the bulge shape parameter and morphological type. The bulges of early-type spirals are characterized by $n \approx 4$ (i.e., they have a de Vaucouleurs radial surface brightness profile) while the bulges of late-type spirals are characterized by $n \approx 1$ (i.e., they have an exponential radial surface brightness profile). This result has been confirmed in various studies (e.g., de Jong 1996; MacArthur et al. 2003; and Möllenhoff 2004).

However, Balcells et al. (2003) performed bulge-disc decomposition of combined space- and ground-based profiles of a sample of S0s and early-type unbarred spirals and found that their bulges have a smaller Sérsic shape parameter than thought before $(\langle n\rangle=1.7 \pm 0.7)$. They claim that the high $n$ values of ground-based profiles are the result of unresolved nuclear components blending with the bulge light because of seeing. We modeled the surface brightness distribution of the sample galaxies by excluding the central region of the light profiles and therefore our results are not sensitive to this effect. Our sample bulges have a mean shape parameter $\langle n\rangle=1.45 \pm 0.15$ $(\langle n\rangle=1.56 \pm 0.16$ if lenses are excluded from the fit $)$. This means that bulges of our SB0 galaxies have almost exponential radial surface brightness profiles which are very similar to those found by Balcells et al. (2003).

We found a strong correlation between the bulge effective radius and the disc scale length in our sample galaxies (Pearson coefficient $r=0.91$, Fig. 3). The mean value varies from $\left\langle r_{\mathrm{e}} / h\right\rangle=0.20 \pm 0.01$ to $\left\langle r_{\mathrm{e}} / h\right\rangle=0.22 \pm 0.02$, depending on whether the lens component is or is not taken into account in the surface brightness fit. These values cannot be compared with the $I$-band ratios found by Graham \& Prieto (1999), MacArthur et al. (2003) and Möllenhoff (2004) for mostly unbarred galaxies, because these recent studies include only few or no early-type spirals and no S0 galaxies. Nevertheless, the $r_{\mathrm{e}} / h$ found for our SB0 galaxies favors the idea that this ratio may be only mildly (MacArthur et al. 2003) and not strongly (Graham \& Prieto 1999; Möllenhoff 2004) correlated with Hubble type, and it possibly increases from late- to early-type disk galaxies.

\subsection{The location of SBO bulges on the fundamental plane}

We collected from the literature the measurements of the central stellar velocity dispersion of all our sample galaxies (Prugniel \& Simien 1997; Fisher 1997; Aguerri et al. 2003; Corsini et al. 2003; Wegner et al. 2003), except for NGC 364. For comparison, we corrected the kinematic data to the equivalent of an aperture of radius $r_{\mathrm{e}} / 8$ following the prescription by Jorgensen et al. (1995), where $r_{\mathrm{e}}$ is the effective radius. The aperture-corrected velocity dispersions are given in Table 1 . We adopted the structural parameters derived in Sect. 3.1 and given in Table 3.

We used the Coma galaxies studied by Scodeggio et al. (1998) to obtain a fundamental plane (hereafter FP) template relation for the unbarred early-type galaxies in the $I$ band. For this purpose we selected only the elliptical galaxies and the bulges of the unbarred S0 galaxies, and we applied the aperture-correction to $r_{\mathrm{e}} / 8$ to their central velocity dispersions. The parameters describing the template FP were derived by assuming the Coma cluster at rest in the CMB reference and $H_{0}=75 \mathrm{~km} \mathrm{~s}^{-1} \mathrm{Mpc}^{-1}$ with the result that

$\log r_{\mathrm{e}}=1.12( \pm 0.10) \log \sigma_{0}+0.33( \pm 0.05) \mu_{\mathrm{e}}-8.68( \pm 0.08)$

by minimizing the square root of the residuals along the $\log r_{\mathrm{e}}$ axis. No statistically significant difference was observed when only elliptical galaxies or only bulges of unbarred S0 galaxies 
Table 5. Relative differences of the structural parameters for the galaxies without a lens fitted to the surface brightness profiles They are defined as $\Delta X=\frac{X_{0}-X_{1}}{X_{0}}$ where $X_{0}$ is the parameter when all components are fitted and $X_{1}$ is the parameter when the lens component is not included in the fit.

\begin{tabular}{lccccccc}
\hline \hline \multicolumn{1}{c}{ Galaxy } & \multicolumn{1}{c}{$\Delta \mu_{\mathrm{e}}$} & \multicolumn{1}{c}{$\Delta r_{\mathrm{e}}$} & \multicolumn{1}{c}{$\Delta n$} & \multicolumn{1}{c}{$\Delta \mu_{0}$} & $\Delta h$ & $\Delta \mu_{\mathrm{b}}$ & \multicolumn{1}{c}{$\Delta r_{\mathrm{b}}$} \\
\multicolumn{1}{c}{$(1)$} & \multicolumn{1}{c}{$(2)$} & \multicolumn{1}{c}{$(3)$} & \multicolumn{1}{c}{$(4)$} & \multicolumn{1}{c}{$(5)$} & \multicolumn{1}{c}{$(6)$} & \multicolumn{1}{c}{$(7)$} & \multicolumn{1}{c}{$(8)$} \\
\hline ESO 139-G009 & -0.01 & 0.07 & 0.20 & 0.05 & 0.27 & -0.03 & 0.04 \\
IC 874 & -0.06 & -0.42 & -0.49 & 0.05 & 0.19 & -0.04 & -0.09 \\
NGC 357 & -0.02 & -0.03 & -0.03 & 0.11 & 0.49 & -0.04 & -0.01 \\
NGC 936 & -0.02 & -0.13 & -0.38 & 0.07 & 0.41 & -0.05 & -0.06 \\
NGC 1440 & -0.03 & -0.39 & -0.58 & 0.03 & 0.17 & -0.08 & -0.13 \\
NGC 3412 & -0.14 & -0.05 & 0.05 & 0.02 & 0.12 & -0.02 & -0.06 \\
NGC 7079 & -0.02 & 0.07 & 0.05 & 0.04 & 0.21 & 0.01 & 0.06 \\
\hline
\end{tabular}

Table 6. Length and strength of the bars in the sample galaxies.

\begin{tabular}{lccccccc}
\hline \hline \multicolumn{1}{c}{ Galaxy } & $\begin{array}{c}r_{\mathrm{b}, 1} \\
(\operatorname{arcsec})\end{array}$ & $\begin{array}{c}r_{\mathrm{b}, 2} \\
(\operatorname{arcsec})\end{array}$ & $\begin{array}{c}r_{\mathrm{b}, 3} \\
(\operatorname{arcsec})\end{array}$ & $\begin{array}{c}r_{\mathrm{b}, 4} \\
(\operatorname{arcsec})\end{array}$ & $\begin{array}{c}r_{\mathrm{b}} \\
(\operatorname{arcsec})\end{array}$ & Ref. & $s_{b}$ \\
\hline ESO 139-G009 & 14.4 & 23.4 & 14.0 & 16.1 & $17.0_{-3.0}^{+6.4}$ & 1 & $0.30 \pm 0.03$ \\
IC 874 & 15.0 & 18.7 & 21.0 & 25.0 & $19.9_{-4.9}^{+5.1}$ & 1 & $0.52 \pm 0.02$ \\
IC 4796 & 19.1 & 20.8 & 20.0 & 23.0 & $20.7_{-1.6}^{+2.3}$ & 2 & $0.38 \pm 0.03$ \\
NGC 357 & 25.4 & 28.9 & 30.9 & 31.0 & $29.1_{-3.7}^{+1.9}$ & 2 & $0.44 \pm 0.02$ \\
NGC 364 & 10.2 & 13.3 & 15.5 & 17.5 & $14.1_{-3.9}^{+3.4}$ & 2 & $0.40 \pm 0.02$ \\
NGC 936 & 46.2 & 50.0 & 46.5 & 69.0 & $53.0_{-6.8}^{+16}$ & 2 & $0.42 \pm 0.01$ \\
NGC 1308 & 14.2 & 13.2 & 9.0 & 13.2 & $12.4_{-3.4}^{+1.8}$ & 1 & $0.44 \pm 0.02$ \\
NGC 1440 & 19.2 & 23.2 & 24.6 & 30.5 & $24.4_{-5.2}^{+6.1}$ & 1 & $0.37 \pm 0.04$ \\
NGC 2950 & 32.2 & 36.5 & 35.6 & 33.7 & $34.5_{-2.3}^{+2.0}$ & 2 & $0.40 \pm 0.03$ \\
NGC 3412 & 34.0 & 28.2 & 30.0 & 32.2 & $31.1_{-2.9}^{+2.9}$ & 1 & $0.34 \pm 0.02$ \\
NGC 3941 & 23.6 & 25.5 & 29.0 & 32.0 & $27.5_{-3.9}^{+4.5}$ & 2 & $0.29 \pm 0.03$ \\
NGC 4340 & 61.5 & 58.0 & 40.5 & 64.0 & $56.0_{-15.5}^{+8.0}$ & 2 & $0.49 \pm 0.01$ \\
NGC 6684 & 48.8 & 46.0 & 40.0 & 42.0 & $44.2_{-4.2}^{+4.6}$ & 2 & $0.42 \pm 0.03$ \\
NGC 7079 & 19.0 & 28.9 & 25.0 & 21.9 & $23.7_{-4.7}^{+5.2}$ & 3 & $0.22 \pm 0.06$ \\
\hline
\end{tabular}

NOTE. Column (2): bar length from photometric decomposition; Col. (3): bar length from the ratio of the intensities in the bar and inter-bar region; Col. (4): bar length from $m=2$ moment phase; Col. (5): bar length from phase of the ellipse fit; Col. (6): mean value of the bar length; Col. (7): list of references for the bar length from Fourier analysis: 1 = Aguerri et al. (2003), 2 = this paper, 3 = Debattista \& Williams (2004); Col. (8): bar strength from Fourier analysis.

are considered. The dispersion around this relation $\sigma=0.098$ and has been measured as the rms scatter in the residuals of $\log r_{\mathrm{e}}$.

Figure 4 shows an edge-on view of the template FP, where we overplotted our SB0 bulges. The bulge of IC 874 is located outside the template FP by more than $3 \sigma$. We find that this is true even adopting the structural parameters derived in Sect. 3.3 by means of a photometric decomposition that does not include the lens component. It should be noted that the location of bulges on the FP does not depend on the choice of a Sérsic law instead of a de Vaucouleurs law for their radial surface brightness profile (Falcón-Barroso et al. 2002).

The FP is related to the virial theorem, which is a consequence of the balance of the kinetic and potential energies of systems in equilibrium. Giant elliptical galaxies do show any significant rotation. The mean stellar rotation is therefore not taken into account in estimating their kinetic energy. This is not the case for fainter elliptical galaxies (Davies et al. 1983) and bulges (Kormendy 1982; Kormendy \& Illingworth 1982) which rotate as rapidly as models of isotropic oblate spheroids (Binney 1978).

Following Prugniel \& Simien (1994), we have investigated the contribution of rotation to the total kinetic energy for the nine SB0 bulges for which the stellar velocity curve has been measured. This contribution is given by

$T \propto\left\langle V^{2}\right\rangle+3\left\langle\sigma^{2}\right\rangle$,

assuming an isotropic tensor of velocity dispersions. For isotropic oblate models the square maximum rotation velocity, $V_{\max }^{2}$, and square central velocity dispersion, $\sigma_{0}^{2}$, are a good 


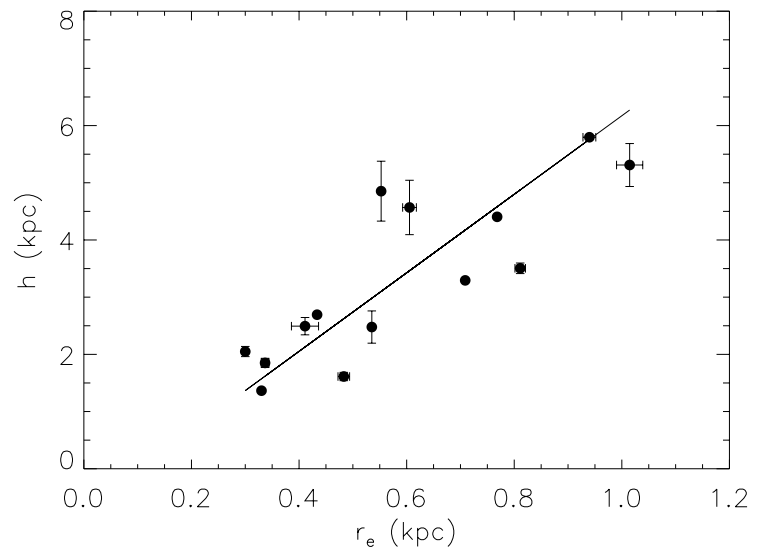

Fig. 3. Disc scale length versus bulge effective radius for the sample galaxies.

approximation of the mass-weighted mean-square rotational velocity, $\left\langle V^{2}\right\rangle$ and velocity dispersion, $\left\langle\sigma^{2}\right\rangle$, respectively (Binney 1980).

We measured the maximum rotation velocity $V_{\max }$ and ellipticity $\epsilon$ of the bulge from the stellar velocity curve and radial ellipticity profile at a radial distance corresponding to $r_{\mathrm{e}}$, respectively. For each galaxy we derived the ratio $\left(V_{\max } / \sigma_{0}\right)^{*}$ of $V_{\max } / \sigma_{0}$ to the value predicted for the observed ellipticity by the oblate models (Table 7). Our SB0 bulges have a weighted mean $\left\langle\left(V_{\max } / \sigma_{0}\right)^{*}\right\rangle=0.94 \pm 0.21$ which is consistent with the early results of Kormendy (1982). Following Kormendy (1982) we conclude that most SB0 bulges rotate as fast as both bulges of unbarred galaxies and oblate spheroid models. This is not the case of the bulges of NGC 1308 and NGC 4340 which are rotating significantly faster than oblate spheroid models and are more similar to the triaxial SB0 bulges studied by Kormendy (1982). The values of $V_{\max } / \sigma_{0}$ and $\left(V_{\max } / \sigma_{0}\right)^{*}$ we obtained for NGC 936, NGC 2950 and NGC 4340 can be compared to those obtained by Kormendy (1982). The agreement is good for NGC 2950 but not for NGC 936 and NGC 4340. We attribute this discrepancy to the different estimate we adopted for the $V_{\max }$ value.

By including the contribution of rotation, the scatter of SB0 bulges with respect to the template FP is reduced by about $20 \%$. This is in agreement with results of Prugniel \& Simien (1994). However, the bulge of IC 874 remains located outside the template FP (Fig. 4).

We discount the possibility that this effect is due to either a disturbed morphology or tidal interaction with nearby companions. IC 874 has been selected to have an undisturbed morphology (see Sect. 2). This is confirmed by the analysis of the surface photometry obtained in Sect. 3. IC 874 has five nearby companions within a projected distance of $500 \mathrm{kpc}$ and a systemic velocity difference of less than $500 \mathrm{~km} \mathrm{~s}^{-1}$ (e.g., Aguerri 1999). We have computed the tidal radius for each of them, finding that shortest one corresponds to ESO 508-G039 and is about $37 \mathrm{kpc}$. Since the optical radius of IC 874 is about $5 \mathrm{kpc}$ (from $D_{25} / 2$ in Table 1) we conclude it does not suffer any tidal interaction. Finally, the location of IC 874 outside of the template FP does not depend on the inclusion of the lens component in the fit of the surface brightness profile.

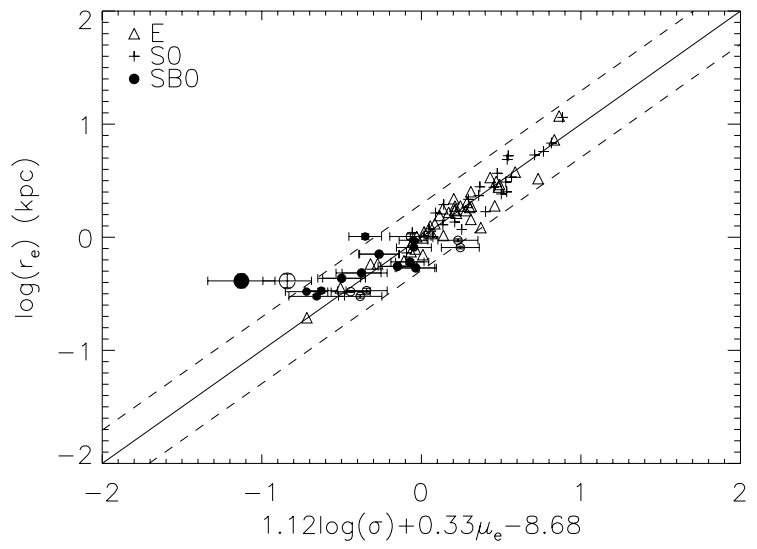

Fig. 4. Edge-on view of the fundamental plane (continuous line) of the Coma elliptical galaxies (triangles) and S0 bulges (open triangles) of Scodeggio et al. (1998). SB0 bulges are indicated by filled circles when rotation is not taken into account to estimate the kinetic energy and by open circles when rotation is included. The dashed lines represent the $3 \sigma$ deviation from the FP. The largest symbol corresponds to IC 874.

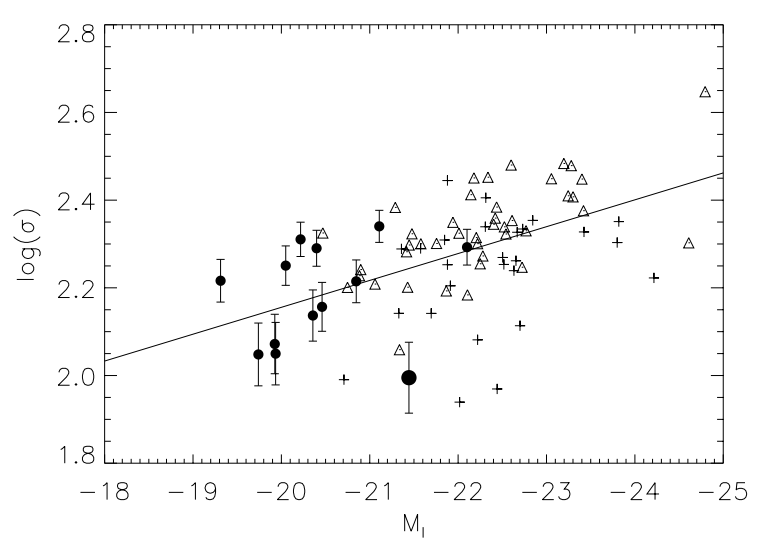

Fig. 5. Ellipticals (triangles) and bulges of S0 (crosses) galaxies from the Coma cluster. The full line represents the FJ relation for bulges of S0 and E galaxies. The full points correspond to the bulges of SB0 galaxies. The largest symbol corresponds to IC 874 .

\subsection{The location of SBO bulges in the Faber-Jackson relation}

One of the projections of the FP is the Faber-Jackson relation (hereafter FJ) which relates the luminosity of elliptical galaxies and S0 bulges to their central velocity dispersion (Faber \& Jackson 1976).

SB0 bulges are consistent with the template FJ relation we built using the same sample of Coma ellipticals and S0 bulges which has been adopted in Sect. 4.3 to obtain the template FP (Fig. 5). Nonetheless, the bulge of IC 874 is a low- $\sigma$ outlier and falls in the region of late-type bulges, which are usually characterized by a lower velocity dispersion or equivalently a higher luminosity with respect to their early-type counterparts (Kormendy \& Kennicutt 2004). This does not depend on whether the lens is included or not in the fit of the surface brightness profile of the galaxy. 
Table 7. Structural parameters of the sample galaxies.

\begin{tabular}{lccccc}
\hline \hline \multicolumn{1}{c}{ Galaxy } & $\begin{array}{c}V_{\max } \\
\left(\mathrm{km} \mathrm{s}^{-1}\right)\end{array}$ & $V_{\max } / \sigma_{0}$ & $\left(V_{\max } / \sigma_{0}\right)^{*}$ & $\epsilon$ & Ref. \\
\multicolumn{1}{c}{$(1)$} & $(2)$ & $(3)$ & $(4)$ & $(5)$ & $(6)$ \\
\hline ESO 139-G009 & $88 \pm 18$ & $0.45 \pm 0.13$ & $1.16 \pm 0.33$ & $0.13 \pm 0.02$ & 1 \\
IC 874 & $44 \pm 3$ & $0.45 \pm 0.20$ & $1.77 \pm 0.79$ & $0.06 \pm 0.01$ & 1 \\
NGC 936 & $78 \pm 10$ & $0.38 \pm 0.05$ & $1.03 \pm 0.13$ & $0.12 \pm 0.01$ & 2 \\
NGC 1308 & $71 \pm 10$ & $0.32 \pm 0.09$ & $1.41 \pm 0.37$ & $0.05 \pm 0.01$ & 1 \\
NGC 1440 & $68 \pm 6$ & $0.35 \pm 0.06$ & $1.05 \pm 0.17$ & $0.10 \pm 0.03$ & 1 \\
NGC 3412 & $31 \pm 6$ & $0.28 \pm 0.18$ & $0.63 \pm 0.40$ & $0.16 \pm 0.05$ & 1 \\
NGC 2950 & $93 \pm 7$ & $0.57 \pm 0.04$ & $0.87 \pm 0.07$ & $0.30 \pm 0.03$ & 3 \\
NGC 3941 & $45 \pm 11$ & $0.33 \pm 0.08$ & $0.81 \pm 0.20$ & $0.14 \pm 0.03$ & 4 \\
NGC 4340 & $35 \pm 9$ & $0.30 \pm 0.08$ & $1.71 \pm 0.43$ & $0.03 \pm 0.01$ & 5 \\
\hline
\end{tabular}

NOTE. Column (2): bulge velocity measured at $r_{\mathrm{e}}$ given by Table 3; Col. (3): ratio of the bulge velocity to central dispersion given in Table 1; Col. (4): ratio of $V_{\max } / \sigma_{0}$ to the value predicted for the observed ellipticity by the oblate models (see Kormendy \& Illingworth 1982); Col. (5): ellipticity of the bulge $\epsilon=1-b / a$ at $r_{\mathrm{e}}$; Col. (6): list of references for the stellar velocity curve: $1=$ Aguerri et al. (2003), $2=$ Kormendy (1983), 3 = Corsini et al. (2003), 4 = Fisher (1997), 5 = Kormendy (1982).

\section{Discussion and conclusions}

The formation of bulges is still an open question and several mechanisms have been proposed (see Wyse et al. 1997, for a review). It has been claimed that bulges formed before discs via hierarchical clustering merging processes (Kauffmann \& White 1993), at the same time as discs during a "monolithic" collapse process (Eggen et al. 1962) or after discs as a results of a secular evolution processes (Kormendy 1979). Furthermore, bulges can experience acquisition events via infall of external material or accretion of satellite galaxies (e.g., Aguerri et al. 2001). Up to now none of these pictures has been able to reproduce all the observed properties of bulges along the Hubble sequence of disc galaxies, leading to the idea that several of the processes outlined in the different scenarios could have played a role in forming a galaxy (Bouwens et al. 1999).

One issue that is not well understood is the influence of bars on bulge formation. Bars are very efficient mechanisms for driving material from the outermost to the innermost regions of spiral galaxies, and produce the formation and/or growth of the bulge component. Recently, Debattista et al. (2004) have studied the bar-buckling instability through a set of high resolution collisionless $N$-body simulations. They conclude that the bar formation drives material to central regions of the disc, building a component which shows an almost exponential surface brightness profile. Although we observed that the SB0 bulges have almost exponential surface brightness profiles, it is difficult to conclude that their assembly is part of the bar formation process. In fact, SB0 bulges have the same shape parameters as bulges of early-type unbarred galaxies (Balcells et al. 2003). This means that it is difficult to photometrically distinguish the influence of the bar on the formation of SB0 bulges. Nevertheless, the fact that bulges of SB0 galaxies have almost exponential surface brightness profiles suggest that they were not formed by violent relaxation in mergers.

In a recent review about the secular evolution of galactic discs, Kormendy \& Kennicutt (2004) discussed the properties of pseudobulges, which are the dense central components in some lenticular and spiral galaxies. In contrast to classical merger-built bulges, pseudobulges are disc-like structures made slowly out of disc gas. We find that the bulges of IC 874, NGC 1380 and NGC 4340 are the most reliable pseudobulges in our sample. The bulges of NGC 1308 and NGC 4340 are more rotation-dominated than classical bulges and oblate spheroid models in the $V_{\max } / \sigma_{0}-\epsilon$ diagram (e.g., Kormendy $\&$ Kennicutt 2004). The bulge of IC 874 is a low- $\sigma$ outlier in the FJ relation and it is outside the $3 \sigma$ band in the FP of elliptical galaxies and S0 bulges. Since IC 874, NGC 1308 and NGC 4340 host the strongest bars of the sample (Table 6) we could speculate that the formation of their bulges has been influenced by the bar. We conclude that the rest of the sample galaxies have classical bulges, which follow the FP and FJ relations traced by bulges of unbarred S0 and elliptical galaxies and rotate as fast as bulges of unbarred galaxies.

Acknowledgements. We would like to thank the anonymous referee for detailed comments which improved the outline and content of the paper. We are most grateful to Victor P. Debattista for making available his $I$-band imaging of NGC 7079 for the present work and for his help in measuring bar length. We acknowledge Luca Ciotti, Cesar González-García, and John Kormendy for useful discussions. E.M.C. thanks the Instituto de Astrofísica de Canarias for the hospitality while this paper was in progress. JALA, NER and CMT have been founded by the Spanish DGES, grant AYA2001-3939. This research has made use of the Lyon Meudon Extragalactic data base (LEDA) and of the NASA/IPAC Extragalactic data base (NED). This paper is based on observations carried out with the New Technology Telescope and the Danish $1.54 \mathrm{~m}$ Telescope (Prop. No. 67.B-0230, 68.B-0329, 69.B-0706, and 70.B-0338) at the European Southern Observatory in La Silla (Chile), with the Italian Telescopio Nazionale Galileo (Prop. AOT-7, TAC_25) operated on the island of La Palma by the Centro Galileo Galilei of the Istituto Nazionale di Astrofisica, and with the Jacobus Kapteyn Telescope operated by the Isaac Newton group on La Palma at the Spanish del Roque de los Muchachos Observatory of the Instituto de Astrofísica de Canarias. 


\section{References}

Aguerri, J. A. L. 1999, A\&A, 351, 43

Aguerri, J. A. L., Debattista, V. P., \& Corsini, E. M. 2003, MNRAS, 338,465

Aguerri, J. A. L., Balcells, M., \& Peletier, R. F. 2001, A\&A, 367, 428

Aguerri, J. A. L., Muñoz-Tuñón, C., Varela, A. M., \& Prieto, M. 2000, A\&A, 361, 841

Andredakis, Y. C., Peletier, R. F., \& Balcells, M. 1995, MNRAS, 275, 874

Athanassoula, E., \& Misiriotis, A. 2002, MNRAS, 330, 35

Balcells, M., Graham, A. W., Domínguez-Palmero, L., \& Peletier, R. F. 2003, ApJ, 582, L79

Baldwin, J. A., \& Stone, R. P. S. 1984, MNRAS, 206, 241

Barnes, J. E., \& Hernquist, L. E. 1991, ApJ, 370, L65

Binney, J. 1978, MNRAS, 183, 501

Binney, J. 1980, MNRAS, 190, 421

Bournaud, F., \& Combes, F. 2002, A\&A, 392, 83

Bouwens, R., Cayón, L., \& Silk, J. 1999, ApJ, 516, 77

Bureau, M., \& Athanassoula, E. 1999, ApJ, 522, 686

Bureau, M., \& Freeman, K. C. 1999, AJ, 118, 126

Burki, G., Rufener, F., Burnet, M., et al. 1995, A\&AS, 112, 383

Caon, N., Capaccioli, M., \& D’Onofrio, M. 1993, MNRAS, 265, 1013

Cardelli, J. A., Clayton, G. C., \& Mathis, J. S. 1989, ApJ, 345, 245

Chung, A., \& Bureau, M. 2004, AJ, 127, 3192

Combes, F., Debbasch, F., Friedli, D., \& Pfenniger, D. 1990, A\&A, 233,82

Combes, F., \& Sanders, R. H. 1981, A\&A, 96, 164

Corsini, E. M., Debattista, V. P., \& Aguerri, J. A. L. 2003, ApJ, 599, L29

Davies, R. L., Efstathiou, G., Fall, S. M., Illingworth, G., \& Schechter, P. L. 1983, ApJ, 266, 41

Debattista, V. P., \& Sellwood, J. A. 2000, ApJ, 543, 704

Debattista, V. P., \& Williams, T. B. 2004, ApJ, 605, 714

Debattista, V. P., Corsini, E. M., \& Aguerri, J. A. L. 2002, MNRAS, 332,65

Debattista, V. P., Carollo, C. M., Mayer, L., \& Moore, B. 2004, ApJ, 604, L93

de Jong, R. S. 1996, A\&A, 313, 45

de Vaucouleurs, G., de Vaucouleurs, A., Corwin, H. G., et al. 1991, Third Reference Catalogue of Bright Galaxies (New York: Springer-Verlag) (RC3)

Duval, M. F., \& Athanassoula, E. 1983, A\&A, 121, 297

Eggen, O. J., Lynden-Bell, D., \& Sandage, A. R. 1962, ApJ, 136, 748

Elmegreen, B. G., Elmegreen, D. M., Chromey, F. R., Hasselbacher, D. A., \& Bissell, B. A. 1996, AJ, 111, 2233

Erwin, P. 2004, A\&A, 415, 941

Erwin, P., Vega Beltrán, J. C., Graham, A. W., \& Beckman, J. E. 2003, ApJ, 597, 929

Eskridge, P. B., Frogel, J. A., Pogge, R. W., et al. 2000, AJ, 119, 536

Faber, S. M., \& Jackson, R. E. 1976, ApJ, 204, 668

Falcón-Barroso, J., Peletier, R. F., \& Balcells, M. 2002, MNRAS, 335, 741

Fathi, K., \& Peletier, R. F. 2003, A\&A, 407, 61

Fisher, D. 1997, AJ, 113, 950

Freeman, K. C. 1966, MNRAS, 133, 47

Freeman, K. C. 1970, ApJ, 160, 811

Friedli, D. 1999, in The Evolution of Galaxies on Cosmological Timescales, ed. J. E. Beckman, \& T. J. Mahoney (San Francisco: ASP), ASP Conf. Ser., 187, 88
Friedli, D., \& Martinet, L. 1993, A\&A, 277, 27

Graham, A. W., \& Prieto, M. 1999, ApJ, 524, L23

Hasan, H., \& Norman, C. 1990, ApJ, 361, 69

Hasan, H., Pfenniger, D., \& Norman, C. 1993, ApJ, 409, 91

Hohl, F. 1971, ApJ, 168, 343

Jorgensen, I., Franx, M., \& Kjaergaard, P. 1995, MNRAS, 276, 1341

Kauffmann, G., \& White, S. D. M. 1993, MNRAS, 261, 921

King, D. L. 1985, La Palma Technical Note, N 31

Kormendy, J. 1993, in Galactic Bulges, ed. H. Dejonghe, \& J. H. Habing (Dordrecht: Kluwer), IAU Symp., 153, 209

Kormendy, J. 1979, ApJ, 227, 714

Kormendy, J. 1982, ApJ, 257, 75

Kormendy, J. 1983, ApJ, 275, 529

Kormendy, J., \& Kennicutt, R. C., Jr. 2004, ARA\&A, 42, 603

Kormendy, J., \& Illingworth, G. 1982, ApJ, 256, 460

MacArthur, L. A., Courteau, S., \& Holtzman, J. A. 2003, ApJ, 582, 689

Möllenhoff, C. 2004, A\&A, 415, 63

Moellenhoff, C., Matthias, M., \& Gerhard, O. E. 1995, A\&A, 301, 359

Odewahn, S. C. 1996, in Barred Galaxies, ed. R. Buta, D. A. Crocker, \& B. G. Elmegreen (San Francisco: ASP), IAU Coll. 157, ASP Conf. Ser., 91, 30

O’Neill, J. K., \& Dubinski, J. 2003, MNRAS, 346, 251

Peng, C. Y., Ho, L. C., Impey, C. D., \& Rix, H. 2002, AJ, 124, 266

Pfenniger, D. 1991, in Dynamics of Disc Galaxies, ed. B. Sundelius (Göteborg: Göteborg University), 191

Pfenniger, D., \& Norman, C. 1990, ApJ, 363, 391

Pohlen, M., Dettmar, R.-J., Lütticke, R., \& Aronica, G. 2002, A\&A, 392, 807

Press, W. H., Teukolsky, S. A., Vetterling, W. T., \& Flannery, B. P. 1992, Numerical Recipes. The Art of Scientific Computing (Cambridge: Cambridge University Press)

Prieto, M., Gottesman, S. T., Aguerri, J. L., \& Varela, A. 1997, AJ, 114,1413

Prieto, M., Aguerri, J. A. L., Varela, A. M., \& Muñoz-Tuñón, C. 2001, A\&A, 367, 405

Prugniel, P., \& Simien, F. 1997, A\&A, 321, 111

Prugniel, P., \& Simien, F. 1994, A\&A, 282, L1

Raha, N., Sellwood, J. A., James, R. A., \& Kahn, F. D. 1991, Nature, 352,411

Schlegel, D. J., Finkbeiner, D. P., \& Davis, M. 1998, ApJ, 500, 525

Scodeggio, M., Gavazzi, G., Belsole, E., Pierini, D., \& Boselli, A. 1998, MNRAS, 301, 1001

Seifert, W., \& Scorza, C. 1996, A\&A, 310, 75

Sersic, J. L. 1968, Atlas de Galaxes Australes (Cordoba: Observatorio Astronomico de Cordoba)

Shen, J., \& Sellwood, J. A. 2004, ApJ, 604, 614

Tremaine, S., \& Weinberg, M. D. 1984, ApJ, 282, L5

Trujillo, I., Aguerri, J. A. L., Cepa, J., \& Gutiérrez, C. M. 2001a, MNRAS, 321, 269

Trujillo, I., Aguerri, J. A. L., Cepa, J., \& Gutiérrez, C. M. 2001b, MNRAS, 328, 977

Valenzuela, O., \& Klypin, A. 2003, MNRAS, 345, 406

Wegner, G., Bernardi, M., Willmer, C. N. A., et al. 2003, AJ, 126, 2268

Wyse, R. F. G., Gilmore, G., \& Franx, M. 1997, ARA\&A, 35, 637 